improve survival rates. These results should be evaluated prospectively in future studies to find the prediction power in the differentiation of flare and sepsis in this group of patients.

\section{THE ROLE OR NEUTROPHIL-LYMPHOCYTE RATIO(NLR), AND OTHER BIOMARKERS (C - REACTIVE PROTEIN CRP, COUNT OF MONOCITES AND LYMPHOCITES) DIFFERENTIATING LUPIC ACTIVITY (FLARE) FROM INFECTION}

1J Aponte, ${ }^{2} \mathrm{~J}$ Carrizosa, ${ }^{3} \mathrm{~A}$ Sanchez, ${ }^{4} \mathrm{M}$ Ospina, ${ }^{1} \mathrm{~A}$ Cartagena, ${ }^{1} \mathrm{C}$ Zapata*, ${ }^{5} \mathrm{R}$ Cervera. ${ }^{1}$ Hospital Universitario de la Samaritana - Universidad de la Sabana, Medicina Interna, Bogota, Colombia; ${ }^{2}$ Universidad del Rosario, Especialista en Cuidado Critico, Bogota, Colombia; ${ }^{3}$ Hospital Universitario de la Samaritana, Cuidado intensivo - Medicina interna, Bogota, Colombia; ${ }^{4}$ Hospital Universitario de la Samaritana, Cuidado intensivo, Bogota, Colombia; ${ }^{5}$ Hospital Clínic de Barcelona, Médico Consultor Senior y Jefe del Servicio de Enfermedades Autoinmunes del Hospital Clínic de Barcelona, Barcelona, Spain

\subsection{6/lupus-2017-000215.202}

Background and aims Systemic lupus erythematosus (SLE) is one of the most prevalent connective tissue diseases, it is commonly associated with an infection being so difficult to differentiate if the systemic inflammatory response is secondary to a bacterial infection, or to the underlying autoimmune activity (FLARE). The aim of this study was to determine the utility of $\mathrm{C}$ reactive protein (CRP), neutrophil-lymphocyte ratio (NLR), count of monocites, and lymphocites in patients with positive cultures and flare.

Methods A retrospective study was conducted, 58 patients with SLE were admitted to the intensive care unit (ICU) at a University Hospital in Bogotá, Colombia, between 2008 and 2017. Bivariate analysis was performed to identify if there was a possible association with positive cultures in patients with (flare)

Results In patients with lupic activity (SLEDAI:8-12) NRL was consistently associated with flare, NRL $>10$ (OR: 17; 95\% CI 2.13 to $136.8, \mathrm{p}=0.007)$, count of lymphocites $<500$ cells/ $\mathrm{mm}^{3}$ was associated with lupic activity (OR: 6.33; 95\% CI 1.30 to $30.7, \mathrm{p}=0.022$ ), in severe lupic activiy de CRP did not show association; one variable consistently associated with positive cultures in the logistic regression model with adequiate prediction parameters: absolute count of monocite $>400 \mathrm{cell} / \mathrm{mm} 3$ (OR: 3.51 ; 95\% CI 1.13 to $10.88, \mathrm{p}=0.029$ ), the others variables NRL, CRP showed no association with positive cultures.

Conclusions The (NRL) $>10$ Could help to differentiate LES activity from infection, leading to early antibiotic therapy, or immunotherapy to improve survival rates. These results should be evaluated prospectively in future studies.

\section{SLE Organ manifestations: clinical and pathogenesis}

\section{EYE MANIFESTATIONS OF LUPUS}

E Abd ElLatif. Alexandria, Egypt

\subsection{6/lupus-2017-000215.203}

Background and aims The body is one unit and the ultimate care of a patient with a multisystem disease such as lupus requires the integrated care of several specialists. Systemic lupus has some potentially blinding ocular complications such as lupus retinopathy. Early detection of these complications by the ophthalmologist can help salvage vision of the patient. Moreover, some cases present first to the ophthalmologist, so an ophthalmologist trained in detecting the ocular manifestations of multisystem diseases can refer the patient promptly to the rheumatologist and help minimise the disease-associated morbidity. The aim of this study was to describe the ocular manifestations of lupus in patients who presented to the main university hospital in Alexandria from July 2014 to March 2016.

Methods A prospective study was conducted and included 128 patients with lupus. A thorough ophthalmic examination was conducted by the author using the slitlamp biomicroscope and a fundus lens

Results Out of the 128 patients, 61 patients had lupus retinopathy at time of presentation or developed it de novo during the period of the study. Thirty two patients had lupus keratopathy. And eighty one patients had dry eye of various degrees of severity, 3 of them culminated into potentially sight threatening corneal ulcers. Communication with the treating rheumatologists was done and an overall $81 \%$ improvement in ocular lupus patients was achieved by the end of the study. One patient lost one eye due to late presentation

Conclusions Lupus is a potentially blinding disease requiring full cooperation between the ophthalmologist and the rheumatologist.

\section{SINGLE CENTRECENTER EXPERIENCE WITH 150 SYSTEMIC LUPUS ERYTHEMATOSUS PATIENTS}

S Tekeoglu, D Temiz Karadag, 0 Ozdemir Isik, A Yazici, A Cefle*. Kocaeli University, Internal Medicine- Rheumatology, Kocaeli, Turkey

\subsection{6/lupus-2017-000215.204}

Background and aims Systemic lupus erythematosus (SLE) is an autoimmune disease with diverse clinical manifestations. Here, we present 150 patients with SLE attending our clinic between January and November 2016.

Methods Demographics, clinics, laboratory findings, Systemic Lupus Erythematous Disease Activity Index (SLEDAI), Systemic Lupus International Clinics (SLICC)/American College of Rheumatology (ACR) damage index scores and treatments were analysed. Diagnosis was confirmed with 1997 ACR or 2012 SLICC classifications. Chi-squared or Fisher's exact tests were used for statistical analysis.

Results General characteristics are presented in Table 1. Clinics are presented in Table 2. Treatments patients ever received are presented in Table 3.

Conclusions SLICC damage was positive in patients receiving pulse steroids (57\%), cyclophosphamide (51\%), rituximab (73\%). In long term, 3 (2\%) patients had pulmonary

Abstract 204 Table 1 General characteristics

\begin{tabular}{ll}
\hline Age (years) & $46 \pm 12.8$ \\
\hline Disease duration (months) & $121.3 \pm 92.4$ (min-max:6-132) \\
Gender (Female/Male) & $139(92.7 \%) / 11(7.3 \%)$ \\
SLEDAI & min-max:0-30, median:1.5 \\
SLICC/ACR damage index & min-max:0-5, median:0 \\
\hline
\end{tabular}




\begin{tabular}{ll} 
Abstract 204 Table 2 & Clinical characteristics ( $\mathrm{n}, \%)$ \\
\hline Muco-cutanous & $108(72 \%)$ \\
\hline Arthritis & $100(66.7 \%)$ \\
Renal disease & $72(48 \%)$ \\
Leukopenia/lymphopenia & $94(62.7 \%)$ \\
Hemolytic anaemia & $13(8.7 \%)$ \\
Thrombocytopenia & $28(18.7 \%)$ \\
Serositis & $30(20 \%)$ \\
Nervous system disease & $11(7.3 \%)$ \\
Anti-phospholipid Syndrome & $24(16 \%)$ \\
\hline
\end{tabular}

Abstract 204 Table 3 Treatment $(n, \%)$

\begin{tabular}{ll}
\hline Steroid/pulse treatment & $149(99.3 \%) / 38(25.3 \%)$ \\
\hline Hydroxycholoroquine & $150(100 \%)$ \\
Azathioprine & $107(71.3 \%)$ \\
Mycophenolate mofetil & $55(36.7 \%)$ \\
Cyclophosphamide (iv) & $45(30 \%) ; 10 \pm 4.5$ cycles \\
Rituximab & $15(10 \%)$ \\
Warfarin & $31(20.7 \%)$ \\
Intravenous immunglobulin (IVIG) & $3(2 \%)$ \\
Plasmapheresis & $2(1.3 \%)$ \\
\hline
\end{tabular}

hypertension, 21 (14\%) had avascular necrosis, 6 (4\%) had malignancy. SLE is an autoimmune disease requiring multi-faceted approach.

\section{ANNEXIN II-BINDING IMMUNOGLOBULIN G LEVEL CORRELATES WITH CLINICAL AND RENAL HISTOLOGICAL DISEASE ACTIVITY IN LUPUS NEPHRITIS}

KF Cheung*, S Yung, M Chau, DYH Yap, TM Chan. The University of Hong Kong, Medicine, Pokfulam, Hong Kong S.A.R

\subsection{6/lupus-2017-000215.205}

Background and aims Annexin II mediates anti-dsDNA antibody binding to mesangial cells and downstream inflammatory and fibrotic processes. We investigated the relationship between annexin II-binding IgG and clinical or histological activity in lupus nephritis.

Methods Serial serum samples from 28 patients with Class III/ $\mathrm{IV} \pm \mathrm{V}$ lupus nephritis were studied. Annexin II-binding IgG level was measured with an in-house ELISA. Glomeruli were isolated from NZBWF1 mice, gene and protein expression of annexin II and its binding protein p11 were investigated by real-time PCR and cytochemical staining respectively. Ultrastructural localization of annexin II was determined by electron microscopy and immunogold staining.

Results Annexin II-binding IgG level was associated with antidsDNA level and disease activity in $42 \%$ of lupus nephritis patients. Annexin II-binding IgG level correlated with Activity Index $(r=0.44, p=0.04)$, leukocyte infiltration score $(r=0.52$, $\mathrm{p}=0.02)$, and karyorrhexis/fibrinoid necrosis score $(\mathrm{r}=0.66$, $\mathrm{p}=0.002$ ) in renal biopsies, and also with the amount of mesangial electron-dense deposit scored semi-quantitatively $(\mathrm{r}=0.63, \mathrm{p}=0.009)$. Glomerular annexin II and $\mathrm{p} 11$ expression increased with disease progression in NZBWF1 mice, and annexin II was found on the surface of mesangial cells and in the mesangial matrix, co-localising with electron-dense deposits.

Conclusions Our data demonstrated an association between annexin II-binding IgG level and clinical/histological disease activity in proliferative lupus nephritis. Co-localization of annexin II with electron-dense deposits suggests a pathogenic role for annexin II.

\section{INCREASED URINARY HEPARANASE LEVELS ARE ASSOCIATED WITH ACTIVE LUPUS NEPHRITIS}

${ }^{1} \mathrm{CS}$ Cho, ${ }^{2} \mathrm{KJ}$ Kim, ${ }^{1} \mathrm{~B}$ In-Woon. ${ }^{7}$ Yeouido St. Mary's Hospital, Rheumatology, Seoul, Republic of Korea; ${ }^{2}$ St. Vincent's Hospital- The Catholic University of Korea, Rheumatology, Suwon, Republic of Korea

\subsection{6/lupus-2017-000215.206}

Background and aims Heparan sulfate in glomerular basement membrane is crucial for charge-selective filtration. Heparanase, an endoglycosidase that cleave heparan sulphate, is reported to be up-regulated in several proteinuric diseases. We investigated the association of urinary heparanase level with renal indices in patients with systemic lupus erythematosus (SLE).

Methods Urinary samples were collected from 76 patients with lupus nephritis (LN; 51 active and 25 inactive), 63 SLE patients without renal involvement and 28 healthy individuals (HC). Heparanase levels were measured by ELISA and normalised by urinary creatinine level $(\mathrm{mU} / \mathrm{mg})$.

Results Urinary heparanase levels were increased in SLE patients than HC $(\mathrm{p}<0.001)$. Patients with active LN had significantly higher urinary heparanase levels compared to patients with inactive $\mathrm{LN}$ and without renal involvement (both $\mathrm{p}<0.001$ ), however, there was no difference between latter groups. Urinary heparanase levels positively correlated with proteinuria (measured by spot urine protein/creatinine ratio) and renal SLEDAI $(\gamma=0.514, p<0.001$ and $\gamma=0.365$, $\mathrm{p}=0.004$, respectively), but inversely with serum C3 $(\gamma=-0.432, p<0.001), \mathrm{C} 4(\gamma=-0.279, \mathrm{p}=0.013)$, and $\mathrm{CH} 50$ levels $(\gamma=-0.336, p=0.003)$. In 39 patients with active $L N$ whose samples were obtained at the time of kidney biopsy, urinary heparanase levels showed positive correlation with activity index $(\gamma=0.409, p=0.011)$, but not with chronicity index $(\mathrm{p}>0.05)$. A cut-off value of $444 \mathrm{mU} / \mathrm{mg}$ predicted presence of active LN with sensitivity of $74.5 \%$ and specificity of $67.1 \%$.

Conclusions Urinary heparanase levels are increased in patients with active LN and reflect the activity of nephritis, indicating that urinary heparanase can serve as useful biomarker for active LN.

\section{SUBCLINICAL DETERIORATION OF LEFT VENTRICULAR DIASTOLIC FUNCTION IN SYSTEMIC LUPUS ERYTHEMATOSUS}

${ }^{1}$ YJ Choi, ${ }^{1}$ WH Yoo, ${ }^{1}$ WS Lee, ${ }^{2} \mathrm{C}$ Lee, ${ }^{2}$ MS Lee. ${ }^{1}$ Chonbuk National University Hospital, Internal Medicine, Jeonju, Republic of Korea; ${ }^{2}$ Wonkwang University Hospital, Internal Medicine, Iksan, Republic of Korea

\subsection{6/lupus-2017-000215.207}

Background and aims Systemic lupus erythematosus (SLE) represents diverse cardiac manifestation, but diastolic dysfunction has been reported infrequently. This study is aimed to 